\title{
(2) OPEN ACCESS \\ Diagnostic value of lymphopaenia and elevated serum ACE in patients with uveitis
}

\author{
Philippine Cotte, ${ }^{1}$ Pierre Pradat, $^{2}$ Laurent Kodjikian (D) , ${ }^{1}$ Yvan Jamilloux, ${ }^{3}$ \\ Pascal Seve (iD) 3,4
}

${ }^{1}$ Ophtalmologie, Hôpital de la Croix-Rousse, Lyon, France ${ }^{2}$ Centre for Clinical Research, Hôpital De La Croix-Rousse, Lyon, France

${ }^{3}$ Internal Medicine, Hôpital de la Croix-Rousse, Lyon, France

${ }^{4}$ Hospices Civils de Lyon, Lyon, France

Correspondence to

Pascal Seve, Service De Médecine Interne, Hôpital de la Croix-Rousse, 103 Grande Rue de la Croix-Rousse, Lyon F-69004, France; pascal.seve@chu-lyon.fr

Received 15 April 2020 Revised 22 July 2020 Accepted 17 August 2020
Check for updates

(C) Author(s) (or their employer(s)) 2020. Re-use permitted under CC BY-NC. No commercial re-use. See rights and permissions. Published by BMJ.

To cite: Cotte $P$, Pradat $P$, Kodjikian L, et al. Br J Ophthalmol Epub ahead of print: [please include Day Month Year]. doi:10.1136/ bjophthalmol-2020-

316563

\section{ABSTRACT}

Aim To evaluate the diagnostic worth of elevated serum ACE (SACE) and lymphopaenia, singly or combined, in diagnosing sarcoid uveitis.

Methods Monocentric retrospective study, on a cohort of 996 adult patients referred to our department between March 2001 and December 2018 for a diagnostic work-up of uveitis. The sensitivity (SE), specificity (Sp), positive predictive value (PPV) and negative predictive value (NPV) of the two biomarkers were calculated in different contexts. Results Eight hundred and sixty-eight patient cases were reviewed. The mean age at uveitis onset was 49.4 $( \pm 18.6)$ years. Of them, 144 patients had a diagnosis of sarcoid uveitis. An elevated SACE had SE of $45.8 \%$, Sp of $88.8 \%$, PPV of $44.9 \%$ and NPV of $89.2 \%$ in diagnosing sarcoid uveitis. For lymphopaenia, SE was $15.3 \%$, Sp was $96.7 \%$, PPV was $47.8 \%$ and NPV was $85.2 \%$. For the combination of elevated SACE and lymphopaenia, SE was $18.9 \%$, Sp was $99.0 \%$, PPV was $73.9 \%$ and NPV was $89.5 \%$. The value of this combination varied according to patient age at diagnosis plus anatomoclinical entities: for patients aged $\leq 50$ years, SE was $31.3 \%$, Sp was $99.7 \%$, PPV was $90.9 \%$ and NPV was $94.3 \%$. For granulomatous uveitis, SE was $26.2 \%$, Sp was $97.3 \%$, PPV was $73.3 \%$ and NPV was $82.5 \%$.

Conclusion A combination of elevated serum ACE and lymphopaenia more convincingly suggests sarcoid uveitis than these investigational tests used alone, especially in patients with granulomatous uveitis, while a lack of these markers corresponds to a high NPV.

Trial registration number NCT03863782.

\section{INTRODUCTION}

Sarcoidosis is a major cause of uveitis worldwide. ${ }^{1}$ An accurate diagnosis of sarcoidosis in uveitis patients has important consequences for managing patients' care and visual outcomes as well as medicinal options. ${ }^{2-5}$ Determining whether an uveitis patient also has sarcoidosis is usually established by combining chest imaging and biochemical measures and then preferably confirmed by biopsy results. ${ }^{6-9}$ The lack of a highly sensitive and specific sarcoidosis screening test in uveitis patients poses a major problem in diagnosing, because undetected sarcoidosis can lead to substantial systemic and ocular morbidity. ${ }^{1}$ Although serum ACE (sACE) is the most commonly used diagnostic and activity biomarker for sarcoidosis, it has a sensitivity varying from $40 \%$ to $83 \% .^{710-13}$ Lymphopaenia, a result of peripheral T-cell depletion, occurs in up to $>50 \%$ of sarcoidosis patients and is associated with a chronic course of the disease. ${ }^{14}$ However, large studies investigating lymphopaenia's value in predicting sarcoid uveitis remain scarce. ${ }^{15-17}$ This study was conducted to investigate the value of lymphopaenia and elevated sACE, singly or in combination, as diagnostic biomarkers of sarcoid uveitis.

\section{PATIENTS AND METHODS}

\section{Patients}

This study concerned a retrospective analysis of the records of 1323 consecutive adult patients with 'uveitis' referred to the department of internal medicine (Lyon University Hospital, Lyon, France) by the department of ophthalmology (Lyon University Hospital, Lyon, France) or by non-hospital ophthalmologists between March 2001 and December 2018. The study excluded: uveitis related to pure ophthalmological entities, diagnosed solely by ophthalmological examination and referred for treatment or for ruling out differential diagnoses $(n=205)$, as well as uveitis occurring in the course of a previously diagnosed disease $(n=122)$. This yielded a cohort of 996 adult uveitis patients.

\section{Diagnostic work-up and definitions}

Patients underwent a standard screening protocol for uveitis, which included a complete blood cell count (CBC), a test, a serological test for syphilis and a chest X-ray. Human leucocyte antigen (HLA)B27 typing was performed in patients with acute anterior uveitis. For cases of either chronic or granulomatous uveitis, a measurement of sACE, a QuantiFERONTB Gold Plus test and a highresolution chest CT (HRCT) were performed. ${ }^{7}$

Diagnostic screening for sarcoidosis included conjunctival or skin biopsies if there were clinically suggestive features involved. Some patients underwent minor salivary gland biopsy, transbronchial lung biopsy, bronchoalveolar lavage (BAL), cerebral MRI or nuclear imaging. This work-up was completed in certain patients by anterior chamber paracentesis (with a PCR test for Herpesvirus, Toxoplasma or RNA16S and sometimes an interleukin-10 measurement), vitreous biopsy and/or lumbar puncture, when appropriate.

Serum ACE levels were considered positive if they were elevated $>1 \mathrm{SD}(\mathrm{SD}=16 \mathrm{IU} / \mathrm{L})$ above the mean reference value for our laboratory (>52 IU/L). Any patients undergoing ACE inhibitor, systemic steroid, immunosuppressive or immunomodulatory therapies were excluded. Concerning ACE inhibitors, the present study is a retrospective analysis of 
a prospectively constituted database from our internal medicine department, where we do not measure ACE levels in patients who use ACE inhibitors. Lymphopaenia was considered to be significant if the lymphocyte count was below our laboratory's reference value $\left(<1.0 \times 10^{9} / \mathrm{L}\right)$. Because children often have higher lymphocyte counts than adults, patients $<18$ years at presentation were excluded from the study cohort.

Throughout this study, the Standardization of Uveitis Nomenclature was used for the anatomical classification of uveitis. $^{18}$

Diagnosis of sarcoidosis was based on the Abad's modified criteria, which include 18-FDG Petscan instead of ${ }^{99} \mathrm{Tc}$ scintigraphy. ${ }^{19}$ Patients were presumed to have sarcoid uveitis if they had at least two out of the following four criteria: typical changes on chest X-ray or CT scan, predominantly CD4 lymphocytosis on BAL fluid analysis, an elevated serum ACE, or 18fluorodeoxyglucose (18-FDG) uptake on scintigraphy. Subjects were judged to have indeterminate sarcoid uveitis when only one criterion was met. Other diagnoses were established according to current international criteria. Whenever the intraocular inflammation could not be assigned to a specific diagnosis, the uveitis was considered to be idiopathic.

Briefly, the diagnostic criteria used were:

- International study group for Behcet's disease criteria ${ }^{20}$;

- Levinson's criteria or global diagnostic criteria for birdshot retinochoroidopathy. ${ }^{21}$ All instances of birdshot retinochoroidopathy were HLA-A29 positive.

- 2010 revised McDonald's criteria for multiple sclerosis $(\mathrm{MS})^{22}$ and Okuda's criteria for radiologically isolated syndromes (RIS). All MS or RIS diagnoses were made conjointly with a neurologist.

- Assessment of SpondyloArthritis International Society (ASAS) criteria for spondyloarthritis. ${ }^{23}{ }^{24}$ HLA-B27-positive patients having acute non-granulomatous anterior uveitis with no clinical and/or radiological features of spondyloarthritis were diagnosed as having HLA-B27-related anterior uveitis;

- revised diagnostic criteria for Vogt Koyanagi Harada's disease $^{25}$;

- Gupta's criteria for diagnosing intraocular tuberculosis. ${ }^{26}$

\section{Data collection}

The study harvested patients' demographic data, follow-up times, plus the following ophthalmological characteristics at diagnosis: anatomical type of uveitis (anterior, intermediate, posterior, panuveitis) plus anterior chamber examination (tonometry, slit lamp, biomicroscopy to assess whether the uveitis was granulomatous or not). We also recorded whether the uveitis was acute or chronic, as well as unilateral or bilateral.

\section{Statistical analysis}

Data were entered into an Excel spreadsheet and analysed with R (R Foundation for Statistical Computing, Vienna, Austria). Categorical variables were reported as $\mathrm{n}(\%)$ and continuous variables as mean $\pm \mathrm{SD}$ (normal distribution) or median and IQR (skewed distribution). For categorical variables, comparisons between groups were performed using the chi-squared test or Fisher's exact test, as appropriate. Continuous variables were compared using the Wilcoxon-Mann-Whitney test. Sensitivity $(\mathrm{SE})$, specificity (Sp), positive predictive value (PPV) and negative predictive value (NPV) were calculated for lymphopaenia and serum ACE. Receiver operating characteristic (ROC) curves were plotted; areas under the curve (AUC) with 95\% CIs were reported. Differences between ROC curves were tested using the method published by Hanley and McNeil. ${ }^{27}$ All tests were twotailed and a $\mathrm{p}$ value of $<0.05$ was considered significant.

\section{RESULTS}

\section{Population characteristics}

Nine hundred and ninety-six adult subjects with undifferentiated uveitis underwent measurement-based screening for sarcoidosis. After excluding 125 patients whose CBC and/or serum ACE measurements were missing plus 3 patients treated with ACE inhibitors, 868 patients were analysed. The characteristics of the study population and final diagnosis are reported in table 1 . The mean age at uveitis onset was $49.4 \pm 18.6$ years; 509 patients $(58.6 \%)$ were female and 689 of the patients $(79.3 \%)$ were of Caucasian descent.

Sarcoid uveitis was diagnosed in 144 subjects. Three hundred and forty-nine patients were classified as indeterminate cases and were included in the other diagnoses group. The mean age at uveitis onset in the sarcoidosis group was $57.3 \pm 16.9$ years. There was a preponderance of female $(58.6 \%)$ and white European patients $(79.4 \%)$, but these characteristics did not differ from the comparison with non-sarcoid uveitis patients $(\mathrm{p}=0.161$ and $p=0.663$, respectively) (table 2 ). Panuveitis and bilateral involvement were more common in sarcoid-associated uveitis $(51.4 \%$ and $77.1 \%$, respectively) compared with patients with nonsarcoid uveitis $(27.1 \%$ and $54.7 \% ; \mathrm{p}<0.001$ and $\mathrm{p}<0.001$, respectively) (table 2). Sarcoidosis was definite (biopsy-proven) in 102 subjects (71\%) and presumed in 42 subjects (29\%). Among those 42 patients, 32 were judged to have sarcoid uveitis on the basis of a positive 18-F-FDG PET/CT scan suggestive of sarcoidosis, 9 had alveolar lymphocytosis, while 1 patient had possible neurosarcoidosis. HCRT was performed in the 144 patients diagnosed with sarcoid uveitis (100\%).

The median sACE level was 51.4 IU/L (IQR 35.3-72.0). In subjects with sarcoidosis, median sACE was $79.0 \mathrm{IU} / \mathrm{L}$ (IQR 54.0-99.5) whereas in subjects without sarcoidosis, median sACE was 47.0 IU/L (IQR 33.1-64.1) $(\mathrm{p}<0.001)$. The sACE was elevated in 147 subjects (16.9\%) and elevated levels were observed in 66 subjects with sarcoid uveitis (true positives, $45.8 \%)$ and in 81 subjects with an alternate diagnosis $(11.2 \%)$

Table 1 Basic characteristics of consecutive patients with a first uveitis attack (data are presented as number of cases and percentages (\%) unless otherwise specified)

\begin{tabular}{|c|c|c|}
\hline & & Total $(n=868)$ \\
\hline \multicolumn{2}{|c|}{ Age (years) at onset of uveitis (mean \pm SD) } & $49.4( \pm 18.6)$ \\
\hline Gender & Females & $509(58.6)$ \\
\hline \multirow[t]{4}{*}{ Anatomical localisation of uveitis } & Anterior & $308(35.5)$ \\
\hline & Intermediate & $96(11.1)$ \\
\hline & Posterior & $194(22.4)$ \\
\hline & Panuveitis & $270(31.1)$ \\
\hline \multirow[t]{5}{*}{ Ethnic group } & White Europeans & $689(79.4)$ \\
\hline & North Africans & $133(15.3)$ \\
\hline & African & $30(3.5)$ \\
\hline & Asian & $13(1.5)$ \\
\hline & Others & $3(0.3)$ \\
\hline Laterality & Bilateral & $507(58.4)$ \\
\hline \multirow[t]{2}{*}{ Chronology } & Acute & $258(29.7)$ \\
\hline & Chronic & $610(70.3)$ \\
\hline Granulomatous & & $247(28.5)$ \\
\hline
\end{tabular}

Cotte $\mathrm{P}$, et al. Br J Ophthalmol 2020;0:1-6. doi:10.1136/bjophthalmol-2020-316563 
Table 2 Comparison between sarcoid uveitis and non-sarcoid uveitis (data are presented as number of cases and percentages (\%) unless otherwise specified)

\begin{tabular}{|c|c|c|c|c|c|}
\hline & & Sarcoid uveitis $(n=144)$ & Other diagnoses $(n=724)$ & Total $(n=868)$ & $\mathbf{P}$ \\
\hline \multicolumn{2}{|c|}{ Age (years) at onset of uveitis (mean $\pm S D$ ) } & $57.3( \pm 16.9)$ & $47.9( \pm 18.5)$ & $49.4( \pm 18.6)$ & $<0.001$ \\
\hline Gender & Females & $92(63.9)$ & $417(57.6)$ & 509 (58.6) & 0.161 \\
\hline \multirow{3}{*}{ Anatomical localisation of uveitis } & Intermediate & $14(9.7)$ & $82(11.3)$ & $96(11.1)$ & \\
\hline & Posterior & $17(11.8)$ & $177(24.4)$ & $194(22.4)$ & \\
\hline & Panuveitis & $74(51.4)$ & $196(27.1)$ & $270(31.1)$ & \\
\hline \multirow{4}{*}{ Ethnic group } & North African & $21(14.6)$ & $112(15.5)$ & $133(15.3)$ & \\
\hline & African & $5(3.5)$ & $25(3.5)$ & $30(3.5)$ & \\
\hline & Asian & $4(2.8)$ & $9(1.2)$ & $13(1.5)$ & \\
\hline & Others & $0(0.0)$ & $3(0.4)$ & $3(0.3)$ & \\
\hline Laterality & Bilateral & $111(77.1)$ & $396(54.7)$ & $507(58.4)$ & $<00.001$ \\
\hline
\end{tabular}

(false positives, 55.1\%) $(\mathrm{p}<0.001)$. Older age appeared somewhat associated with elevated sACE although the difference did not reach statistical significance (Wilcoxon-Mann-Whitney test, $\mathrm{p}=0.058$ ). Subjects were more likely to have elevated sACE if they had intermediate uveitis or panuveitis $\left(\chi^{2}\right.$ test, $\left.p=0.007\right)$, or granulomatous uveitis (Fisher's exact test, $p<0.001$ ), or if they were of North African or Afro-Caribbean origin (Fisher's exact test, $\mathrm{p}=0.012$ ). The most common diagnosis in the false-positive group was idiopathic uveitis $(n=36,44.4 \%)$. Other diagnoses in the false-positive group included: HLA-B27-related anterior uveitis $(n=5,6.2 \%)$, infectious uveitis $(n=21,25.9 \%$; syphilis, tuberculosis, herpes simplex virus and Lyme's disease). There were also six cases of birdshot retinochoroidopathy, one Behcet's disease, one Vogt Koyanagi Harada's disease and two cases of ocular lymphoma.

In subjects with sarcoid uveitis, the median lymphocyte count was $1.50 \times 10^{9} / \mathrm{L}$ (IQR 1.10-1.90) whereas in subjects without sarcoid uveitis, the median lymphocyte count was $2.00 \times 10^{9} / \mathrm{L}$ (IQR 1.52-2.54) $(\mathrm{p}<0.001)$. Overall, lymphopaenia was observed in 46 subjects (5.3\%). It was observed in 22 subjects with sarcoid uveitis (15.3\%) (true positives, $47.8 \%$ ), plus in 24 subjects having an alternate diagnosis (3.3\%) (false positives, $52.2 \%)(\mathrm{p}<0.001)$. Granulomatous uveitis $(\mathrm{p}=0.006)$ and older age $(p<0.001)$ were also associated with lymphopaenia. The most common diagnosis in the false-positive group was idiopathic uveitis $(n=13,54.2 \%)$. Other diagnoses in the falsepositive group included HLA-B27-related anterior uveitis $(\mathrm{n}=2$, $8.3 \%)$ and infectious uveitis $(n=4,16.6 \%$; herpes simplex virus and toxoplasmosis). There were also three birdshot retinochoroidopathy and two ocular lymphoma diagnoses.

The number of patients in each category is shown in table 3.

\section{RELIABILITY OF SERUM ACE AND LYMPHOPAENIA IN} SYSTEMIC SARCOIDOSIS DIAGNOSING

Using the standard cut-off of $68 \mathrm{IU} / \mathrm{L}, \mathrm{SE}$ of sACE in the diagnosis of sarcoid uveitis was $45.8 \%$, Sp was $88.8 \%$, PPV was $44.9 \%$ and NPV was $89.2 \%$ (table 4). AUC was 0.778 (95\% CI 0.72 to $0.836, p<0.001$ ) (figure 1 ). Based on this ROC curve, the optimal cut-off for sACE to maximise both specificity and sensitivity in adult subjects was $61.2 \mathrm{IU} / \mathrm{L}$, resulting in SE of $72.3 \%$ and
Table 3 Number of patients in each category

\begin{tabular}{llc}
\hline & Sarcoid uveitis & Other diagnoses \\
\hline Lymph 1+ECA 1 & 17 & 6 \\
Lymph 1+ECA 0 & 5 & 18 \\
Lymph 0+ECA 1 & 49 & 75 \\
Lymph 0+ECA 0 & 73 & 625
\end{tabular}

With: Lymph 1=lymphopaenia, Lymph $0=$ normal lymphocyte count, ECA 1=elevated ECA and ECA $0=$ normal ECA.

Table 4 Summary of test characteristics of lymphopaenia, elevated ACE and the combination of both for the diagnosis of sarcoid uveitis (data are presented as percentages (\%))

\begin{tabular}{lllll}
\hline Biological parameters & Sensitivity & Specificity & PPV & NPV \\
\hline Elevated serum ACE & 45.8 & 88.8 & 44.9 & 89.2 \\
Lymphopaenia & 15.3 & 96.7 & 47.8 & 85.2 \\
Association of both parameters & 18.9 & 99.0 & 73.9 & 89.5 \\
\hline
\end{tabular}

ACE, angiotensin-converting enzyme; NPV, negative predictive value; PPV, positive predictive value.

Sp of $72.5 \%$. The sensitivity for lymphopaenia in the diagnosis of sarcoid uveitis was $15.3 \%$, Sp was $96.7 \%$, PPV was $47.8 \%$ and NPV was $85.2 \%$. AUC was 0.713 (95\% CI, 0.655 to 0.771 , $\mathrm{p}<0.001$ ) (figure 2). Based on this ROC curve, the optimal cutoff for lymphopaenia to maximise both specificity and sensitivity in adult subjects was $1.74 \mathrm{G} / \mathrm{L}$, resulting in a SE of $63.7 \%$ and a Sp of $65.3 \%$.

The sensitivity for the combination of elevated sACE and lymphopaenia in the diagnosis of sarcoid uveitis was $18.9 \%, \mathrm{Sp}$ was $99.0 \%$, PPV was $73.9 \%$ and NPV was $89.5 \%$. This is quite similar to lymphopaenia alone where the difference does not reach statistical significance (Hanley and McNeil test, $\mathrm{p}=0.431$ ) but slightly different from elevated sACE alone where the difference reaches statistical significance $(p=0.026)$. When solely examining the biopsy-proven sarcoid uveitis group, SE for the 


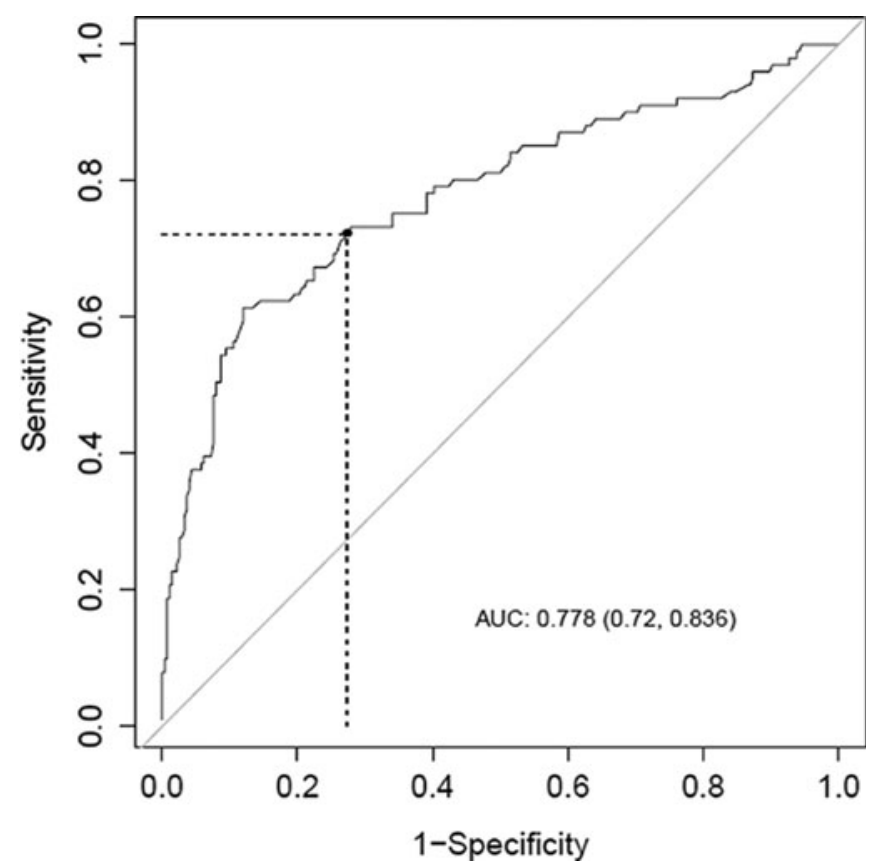

Figure 1 Receiver operating characteristic curve for elevated serum ACE in adult sarcoid uveitis. ACE, angiotensin-convertingenzyme.

combination of elevated sACE and lymphopaenia was $23.7 \%$, Sp was $98.6 \%$, PPV was $60.9 \%$ and NPV was $93.6 \%$.

The sensitivity for the combination of lymphopaenia and/or elevated sACE in the diagnosis of sarcoid uveitis was $49.3 \%, \mathrm{Sp}$ was $86.3 \%$, PPV was $41.8 \%$ and NPV was $89.5 \%$.

When using the optimal cut-off determined through the ROC curves (ie, $1.74 \mathrm{~g} / \mathrm{L}$ for lymphopaenia and 61.2 IU/L for sACE), SE for elevated sACE in the diagnosis of sarcoid uveitis was $70.5 \%$, Sp was $72.4 \%$, PPV was $44.0 \%$, and NPV was $88.8 \%$. The SE for lymphopaenia in the diagnosis of sarcoid uveitis was

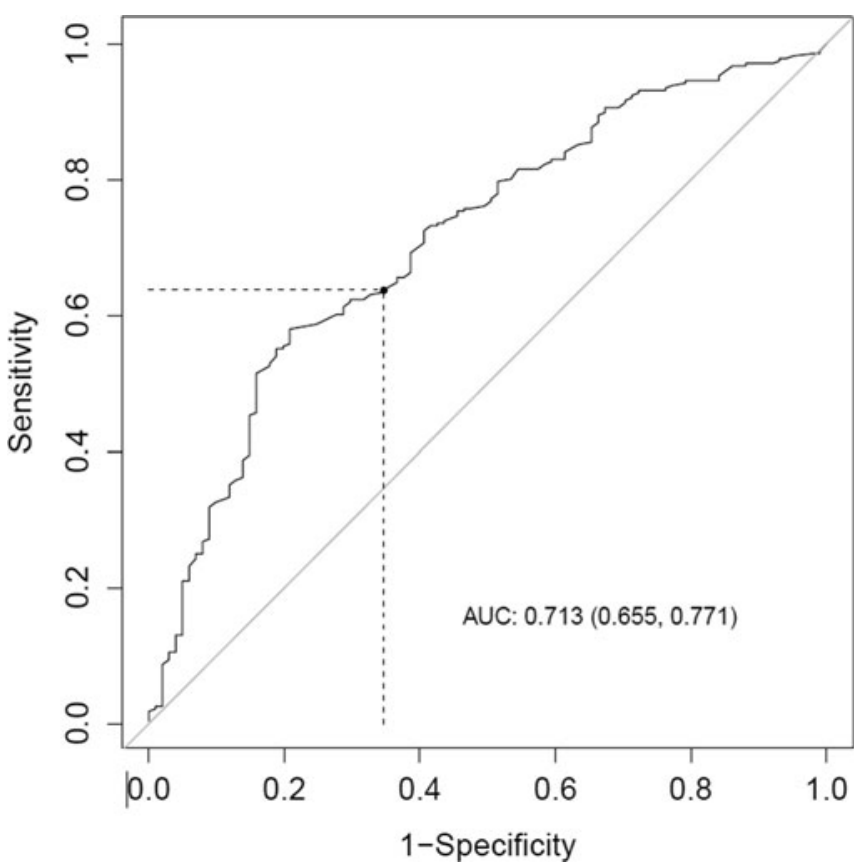

Figure 2 Receiver operating characteristic curve for lymphopaenia in adult sarcoid uveitis.
$62.2 \%$, Sp was $63.4 \%$, PPV was $31.3 \%$ and NPV was $86.7 \%$. The sensitivity for the combination of elevated sACE and lymphopaenia in the diagnosis of sarcoid uveitis was $49.5 \%$, Sp was $89.9 \%$, PPV was $64.1 \%$ and NPV was $82.9 \%$.

\section{COMPARISON OF RELIABILITY INDICES BY DEMOGRAPHICS AND ANATOMIC TYPES}

When focusing on patients' ages, for patients aged $\leq 50$ years, SE for the combination of elevated sACE and lymphopaenia was $31.3 \%$, Sp was $99.7 \%$, PPV was $90.9 \%$ and NPV was $94.3 \%$. For patients aged $>50$ years, SE for the combination of elevated sACE and lymphopaenia was $12.1 \%$, Sp was $73.7 \%$, PPV was $58.3 \%$ and NPV was 21.5\% (Hanley and McNeil test, $p=0.230$ ). Thus, the difference does not reach statistical significance.

When studying anatomoclinical entities, for granulomatous uveitis SE for the combination was 26.2\%, Sp was 97.3\%, PPV was $73.3 \%$ and NPV was $82.5 \%$; while for bilateral uveitis SE for the combination was $20.3 \%$, Sp was $98.3 \%$, PPV was $77.8 \%$ and NPV was $86.2 \%$.

\section{DISCUSSION}

This study covers a large number of subjects presenting with unexplained uveitis in whom serum ACE and lymphocyte counts were both routinely measured.

The principle of such a biomarker combination was first proposed for sACE and lysozyme levels by Baarsma et al in 1987, while studying 221 patients with ocular sarcoidosis. ${ }^{28}$

Based on a study of 191 patients with a first uveitis episode, Groen-Hakan et al found that lymphopaenia was strongly associated with a diagnosis of sarcoidosis, with an optimal cut-off of $1.47 \times 10^{9} / \mathrm{L}$, a strong sensitivity of $75 \%$ and a specificity of $77 \% .{ }^{15}$ Their study excluded patients undergoing immunosuppressive therapies. In our study, we found a low sensitivity of $15.3 \%$ which may be explained by the fact that we used a lower cut-off of $1.0 \times 10^{9} / \mathrm{L}$. Our cut-off is the same as that used by Jones et al who found a sensitivity of $31.6 \%$ in their study based on 112 patients with sarcoid uveitis. ${ }^{16}$

Regarding the serum ACE elevation, Niederer et al found a high sensitivity of $78.1 \%$ whereas our study found $45.8 \% .^{13}$ These authors found a specificity of $90.0 \%$, quite similar to our study $(88.8 \%)$. They also found very high NPV of $97.0 \%$ for normal ACE in subjects presenting with undifferentiated uveitis, whereas our NPV was $89.2 \%$. However, the PPV found by Niederer et al was only $43.6 \%$ and our study's PPV for ACE was not much better (44.9\%). This difference may relate to different diagnostic criteria and different levels of aggressiveness in pursuing the sarcoidosis diagnosis. Moreover, in Sims and Niederer study, the HRCT was not routinely performed, and more borderline cases may have been picked-up in our study, thus explaining the lower sensitivity of sACE.

Predictive values indicate the probability of disease in a patient with a positive test result (PPV) or the probability of no disease in a patient if the test is negative (NPV). Since conventional diagnostic tests have low PPV values in diagnosing sarcoid uveitis, a search for a more sensitive and specific diagnostic test is warranted.

Our study showed that combining elevated serum ACE and lymphopaenia makes it possible to enhance specificity to $99.0 \%$, PPV to $73.9 \%$ and NPV to $89.5 \%$ for diagnosing sarcoid uveitis. This means that if both parameters are present, sarcoid uveitis is present in nearly three quarters of all cases. On the other hand, if both parameters are absent, one might miss diagnosing sarcoid 
uveitis in about $10 \%$ of all cases. However, based on our data, $50.7 \%$ of sarcoidosis patients will have negative sACE and no lymphopaenia; hence, the clinical picture remains the cornerstone of sarcoidosis diagnosis. Our study also shows that if the combination of both parameters occurs in patients aged $<50$ years old, sensitivity increases to $31.3 \%$, specificity reaches 99.7\%, PPV 90.9\% and NPV 94.3\%. Moreover, when the uveitis is granulomatous, sensitivity increases to $26.2 \%$, specificity reaches $97.3 \%$, PPV $73.3 \%$ and NPV $82.5 \%$.

The strengths of our study were, first, that HRCT was frequently performed since it was performed in the 144 patients diagnosed with sarcoid uveitis. In previous studies, the HRCTwas performed at physician's discretion. ${ }^{13}$ Several studies have demonstrated the improved sensitivity of this examination over chest X-rays. ${ }^{29}$ Niederer and Sims established the usefulness of screening investigations for systemic sarcoidosis in 709 subjects presenting with undifferentiated uveitis and found a sensitivity and specificity highest for chest CT than chest X-ray $(98 \%$ and $100 \%$ vs $57.6 \%$ and $100 \%$, respectively). ${ }^{17}$ Moreover, Kaiser et al showed that chest CT performed in older female patients with chronic uveitis showed mediastinal lymphadenopathy and other lesions suggestive of sarcoidosis in 17 patients (57\%). ${ }^{30}$ Second, we did not apply the International Workshop on Ocular Sarcoidosis (IWOS) 2009 criteria, since they have been called into question. Indeed, a study by Acharya et al showed that IWOS criteria had low sensitivities in diagnosing ocular sarcoidosis, with many suspected sarcoidosis patients who did not fit into the classification system. ${ }^{31}$ They found that although the IWOS clinical and investigational signs were significantly more often present in ocular sarcoidosis cases compared with control groups, laboratory investigations had low sensitivities, ranging from 5\% for an abnormal liver enzyme test to $70 \%$ for anergy. Moreover, $37 \%$ of patients with suspected ocular sarcoidosis did not meet IWOS criteria. In the recently revised IWOS criteria, Mochizuki et al have changed the diagnostic protocol ${ }^{32}$ : the following four items were adopted by two-thirds of the panel members and instated as IWOS consensus agreement: (a) lymphopaenia, (b) elevated CD4/CD8 ratio (>3.5) in BAL fluid, (c) parenchymal lung changes consistent with sarcoidosis as determined by pulmonologists or radiologists and (d) abnormal label uptake on $67 \mathrm{Ga}$ scintigraphy or $18 \mathrm{~F}$-fluorodeoxyglucose PET imaging. However, this study was composed of a question-based survey and consensus meeting, based on personal experience of panel members.

Finally, our study has some limitations. First, this study is retrospective and monocentric, and includes an indication bias because diagnostic investigations were not performed in all cases and were performed at the discretion of the physicians, leading to a difference in diagnostic criteria making our study more difficult to compare with other studies. Moreover, we had to deal with missing data. Second, since our centre is a tertiary referral centre for uveitis management, some patients already had a long history of uveitis before being referred to our department and thus might differ from daily-care patients. As a result, this can make an important difference as sACE is often high and lymphocytes low during the acute phase, while they are not later in the disease course. However, this point made it possible for the study to include patients with lengthy follow-up after initial diagnosis. Third, patients in this study were referred to internal medicine practitioners by ophthalmologists with no precise pre-established criteria, beyond having an ophthalmological presentation suggestive of systemic disease. This could bias patient selection towards over-representation of systemic diseases. Finally, another limitation of our study relies in the use of different diagnostic criteria making it difficult to compare with previous studies.

\section{CONCLUSION}

Our study indicates that the combination of lymphopaenia and elevated serum ACE can serve as a useful biomarker for diagnosing sarcoidosis in uveitis patients, demonstrating a better overall diagnostic performance than solely that of serum ACE levels or lymphopaenia alone. This holds particularly true for young patients and those suffering from granulomatous uveitis.

Contributors PC and PS designed this study, collected the data and wrote the manuscript. PP performed the statistical analysis.

Funding This research received no specific grant from any funding agency in the public, commercial or not-for-profit sectors.

\section{Competing interests None declared.}

Ethics approval This study was conducted with the approval of the institutional review board of Hospices Civils de Lyon.

Provenance and peer review Not commissioned; externally peer reviewed.

Data availability statement All data relevant to the study are included in the article or uploaded as supplemental information.

Open access This is an open access article distributed in accordance with the Creative Commons Attribution Non Commercial (CC BY-NC 4.0) license, which permits others to distribute, remix, adapt, build upon this work non-commercially, and license their derivative works on different terms, provided the original work is properly cited, appropriate credit is given, any changes made indicated, and the use is noncommercial. See: http://creativecommons.org/licenses/by-nc/4.0/.

\section{ORCID iDs}

Laurent Kodjikian http://orcid.org/0000-0001-7568-269X

Pascal Seve http://orcid.org/0000-0003-3070-3842

\section{REFERENCES}

1 Jamilloux Y, Kodjikian L, Broussolle C, et al. Sarcoidosis and uveitis. Autoimmun Rev 2014;13:840-9.

2 Bodaghi B, Cassoux N, Wechsler B, et al. Chronic severe uveitis: etiology and visual outcome in 927 patients from a single center. Medicine (Baltimore) 2001;80:263-70.

3 Barisani-Asenbauer T, Maca SM, Mejdoubi L, et al. Uveitis - a rare disease often associated with systemic diseases and infections - a systematic review of 2619 patients. Orphanet J Rare Dis 2012;7:57.

4 Lee CS, Randhawa S, Lee AY, et al. Patterns of laboratory testing utilization among uveitis specialists. Am J Ophthalmol 2016;170:161-7.

5 Valeyre D, Prasse A, Nunes H, et al. Sarcoidosis. Lancet 2014:383:1155-67.

6 Suttorp-Schulten MS, Rothova A. The possible impact of uveitis in blindness: a literature survey. Br J Ophthalmol 1996:80:844-8.

7 de Parisot A, Kodjikian L, Errera $\mathrm{M}-\mathrm{H}$, et al. Randomized controlled trial evaluating a standardized strategy for uveitis etiologic diagnosis (ULISSE). Am J Ophthalmol 2017;178:176-85

8 Hadjadj J, Dechartres A, Chapron T, et al. Relevance of diagnostic investigations in patients with uveitis: retrospective cohort study on 300 patients. Autoimmun Rev 2017:16:504-11.

9 Clement DS, Postma G, Rothova A, et al. Intraocular sarcoidosis: association of clinical characteristics of uveitis with positive chest high-resolution computed tomography findings. Br J Ophthalmol 2010;94:219-22.

10 Birnbaum AD, Oh FS, Chakrabarti A, et al. Clinical features and diagnostic evaluation of biopsy-proven ocular sarcoidosis. Arch Ophthalmol 2011;129:409-13.

11 Grumet $\mathrm{P}$, Kodjikian $\mathrm{L}$, de parisot $\mathrm{A}$, et al. Contribution of diagnostic tests for the etiological assessment of uveitis, data from the ULISSE study (Uveitis: clinical and medicoeconomic evaluation of a standardized strategy of the etiological diagnosis). Autoimmun Rev 2018;17:331-43.

12 Sahin 0, Ziaei A, Karaismailoğlu E, et al. The serum angiotensin converting enzyme and lysozyme levels in patients with ocular involvement of autoimmune and infectious diseases. BMC Ophthalmol 2016:16:19.

13 Niederer RL, Al-Janabi A, Lightman SL, et al. Serum angiotensin-converting enzyme has a high negative predictive value in the investigation for systemic sarcoidosis. Am J Ophthalmol 2019;201:89.

14 Sweiss NJ, Salloum R, Ghandi S, et al. Significant CD4, CD8, and CD19 lymphopenia in peripheral blood of sarcoidosis patients correlates with severe disease manifestations. Plos One 2010:5:e9088. 
15 Groen-Hakan F, Eurelings L, Rothova A, et al. Lymphopaenia as a predictor of sarcoidosis in patients with a first episode of uveitis. Br J Ophthalmol 2019;103:1296-300.

16 Jones NP, Tsierkezou L, Patton N. Lymphopenia as a predictor of sarcoidosis in patients with uveitis. Br J Ophthalmol 2016;100:1393-6.

17 Niederer RL, Sims JL. Utility of screening investigations for systemic sarcoidosis in undifferentiated uveitis. Am J Ophthalmol 2019;206:149-53.

18 Jabs DA, Nussenblatt RB, Rosenbaum JT, et al. Standardization of uveitis nomenclature for reporting clinical data. results of the first international workshop. Am J Ophthalmol 2005; 140:509-16.

19 Abad S, Meyssonier V, Allali J, et al. Association of peripheral multifocal choroiditis with sarcoidosis: a study of thirty-seven patients. Arthritis Rheum 2004;51:974-82.

20 Criteria for diagnosis of Behçet's disease. International Study Group for Behçet's Disease. Criteria for diagnosis of Behçet's disease. International study group for Behçet's disease. Lancet 1990;335:1078-80.

21 Levinson RD, Brezin A, Rothova A, et al. Research criteria for the diagnosis of birdshot chorioretinopathy: results of an international consensus conference. Am J Ophthalmol 2006;141:185-7.

22 Polman CH, Reingold SC, Banwell B, et al. Diagnostic criteria for multiple sclerosis: 2010 revisions to the McDonald criteria. Ann Neurol 2011;69:292-302.

23 Rudwaleit M, van der Heijde D, Landewé R, et al. The development of assessment of Spondylo Arthritis International Society classification criteria for axial spondyloarthritis (part II): validation and final selection. Ann Rheum Dis 2009;68:777-83.
24 Rudwaleit M, van der Heijde D, Landewé R, et al. The assessment of spondyloArthritis international society classification criteria for peripheral spondyloarthritis and for spondyloarthritis in general. Ann Rheum Dis 2011;70:25-31.

25 Read RW, Holland GN, Rao NA, et al. Revised diagnostic criteria for Vogt-KoyanagiHarada disease: report of an international committee on nomenclature. Am J Ophthalmol 2001;131:647-52.

26 Gupta V, Gupta A, Rao NA. Intraocular tuberculosis: an update. Surv Ophthalmol 2007:52:561-87.

27 McNeil BJ, Hanley JA. Statistical approaches to the analysis of receiver operating characteristic (ROC) curves. Med Decis Making 1984;4:137-50.

28 Baarsma GS, La Hey E, Glasius E, et al. The predictive value of serum angiotensin converting enzyme and lysozyme levels in the diagnosis of ocular sarcoidosis. Am J Ophthalmol 1987;104:211-7.

29 Sève $P$, Cacoub P, Bodaghi B, et al. Uveitis: diagnostic work-up. A literature review and recommendations from an expert committee. Autoimmun Rev 2017; 16:1254-64.

30 Kaiser PK, Lowder CY, Sullivan P, et al. Chest computerized tomography in the evaluation of uveitis in elderly women. Am J Ophthalmol 2002;133:499-505.

31 Acharya NR, Browne EN, Rao N, et al. Distinguishing features of ocular sarcoidosis in an international cohort of uveitis patients. Ophthalmology 2018;125:119-26.

32 Mochizuki M, Smith JR, Takase H, et al. Revised criteria of International Workshop on Ocular Sarcoidosis (IWOS) for the diagnosis of ocular sarcoidosis. Br J Ophthalmol 2019;103:1418-22. 